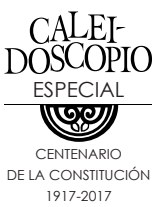

\title{
Cien años de la Constitución de 1917. Representación y participación política de las mujeres en México
}

One hundred years of the Constitution of 1917. Representation and political participation of women in Mexico

BRISA HERMINIA CAMPOS ACEVES'

JUAN PABLO CARRANZA SALAS²

\section{RESUMEN}

El presente trabajo tiene como objetivo responder cuál ha sido la evolución de la participación política de la mujer en México a partir de la reforma al artículo $34^{\circ}$ de la Constitución Política de los Estados Unidos Mexicanos, la cual le permitió votar y ser votada por primera vez, esto a partir de cien años de promulgada. El documento parte de una breve contextualización del constitucionalismo mexicano; posteriormente, describe el acceso a la vida política del país a través del voto de las mujeres en la década de 1950; para continuar con la evolución de las cuotas de género a partir de 1990, con el objetivo de lograr la paridad de género, la cual se ve plasmada en la última reforma constitucional, realizada al artículo $41^{\circ}$ de la Constitución en el 2014.

Palabras clave: Constitución de 1917, participación política de las mujeres, acción afirmativa, paridad de género.

1 Universidad Autónoma de Aguascalientes.

2 Universidad Autónoma de Aguascalientes. 
The present work aims to answer what has been the evolution of the political participation of women in Mexico since the reform of the Article 34 of the Political Constitution of Mexico, which allowed them for the first time to vote and be voted on, after one hundred years of its promulgation. The document starts from a brief contextualization of Mexican constitutionalism; it later describes the access to the political life of the country through the women's vote in the fifties, later on as the evolution of gender quotas from the nineties, with the aim of achieving gender parity, that is reflected in the last constitutional reform made to the Article 41 of the Constitution in 2014.

Keywords: Constitution of 1917, political participation of women, affirmative action, gender parity.

Generalmente, se entiende que una Constitución es un marco normativo orientado a la organización de los poderes de un estado o, como refiere Michelangelo Bovero (2006): "es el ordenamiento de las magistraturas y poderes de un estado cualquiera" (Bovero, 2006: 16-24, en Salazar, 2015: 1930). En el ámbito de la Constitución, la relación entre poder y derecho es muy estrecha: es la organización de los poderes la que da forma a la Constitución y esta última, entonces, no es otra cosa que la expresión normativa de dicha articulación (Salazar, 2015: 1931).

En la modernidad, el concepto de Constitución adquirió un significado preciso referido a un contenido determinado, al menos en el contexto occidental. La definición del concepto de Constitución quedó plasmada en el artículo 16 de la Declaración de los Derechos del Hombre y del Ciudadano de 1789: "Una sociedad en la que la garantía de los derechos no está asegurada, ni la separación de poderes definida, no tiene Constitución" (Salazar, 2015: 1934). Se considera que el origen de la actual Constitución Política de los Estados Unidos Mexicanos nace a partir de la conocida Decena Trágica, con la obligación a renunciar a la presidencia por parte de Francisco I. Madero y su posterior muerte, la toma de la presidencia en forma irregular por Victoriano Huerta, su desconocimiento como presidente por Venustiano Carranza y los intentos de acuerdos entre los líderes revolucionarios por generar acuerdos de paz y organización en el país. 
Carranza se materializó como un auténtico defensor de la Constitución de 1857 (de ideología liberal) y más tarde, con el Plan de Guadalupe, fundamentó el movimiento constitucionalista. En este recorrido hay una lógica irrefragable, como lo afirma el jurista Ulises Schmill: "en primer lugar, estamos ante un acto de desconocimiento o invalidez y, en segundo lugar, ante la fijación de la finalidad fundamental del orden revolucionario, a saber, la restauración del orden constitucional" (López, 2017). La Constitución promulgada el 5 de febrero de 1917 fue la primera en la historia en incluir los derechos sociales, marcando de esta forma un antecedente para el resto del mundo, lo que le otorgó el reconocimiento de "la primer Constitución social del siglo xx" (Madrid, 2016), en la cual, sus principales aportaciones son: la no reelección del presidente; las garantías individuales; el énfasis en la división de poderes en ejecutivo, legislativo y federal; que dejara de existir la figura de vicepresidente, al tiempo que se le daba una mayor soberanía a todos los estados de la República, y que gracias a estas leyes, los ciudadanos podrían disfrutar de derechos y garantías (H. Ayuntamiento de Coatzacoalcos, 2018).

Por su parte, en todos los frentes de lucha están las mujeres: en el militar, participando como soldados rasos o como oficiales (coronelas y capitanas), y haciendo funciones de inteligencia; mujeres desarrollando propaganda contra la dictadura de Porfirio Díaz y contra su reelección, denunciando la situación de miseria de campesinos y peones; otras organizadas en el Partido Liberal Mexicano, pauta para las transformaciones sociales plasmadas en la Constitución (Girón, González y Jiménez, 2008: 39); otras más, promotoras activas del movimiento constitucionalista; y muchas más preocupadas por el reconocimiento de la igualdad entre mujeres y hombres, como la propuesta de incluir los derechos políticos de las mujeres que, dicho sea de paso, no tuvo eco en el Constituyente del 17 (López, 2017).

El presente artículo busca describir la evolución de las reformas constitucionales en materia de reconocimiento de los derechos y participación política de las mujeres. En una primera instancia, identificando el momento de reconocimiento de los derechos políticos de las mujeres, principalmente el otorgamiento al voto y ser votadas; en un segundo momento, con la exposición de la asignación y evolución de las cuotas de género como acciones afirmativas que promueven la participación política de las mujeres; finalmente, al presentar la reforma constitucional del 2014, donde se obliga a los partidos políticos a

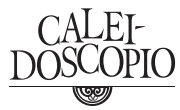


asegurar la paridad de género en las listas de candidatos, así como su impacto en las elecciones federales del 2015.

\section{EL VOTO: DERECHO DE LAS MUJERES}

Los filósofos de la Ilustración consideraban a la razón y a la igualdad como cualidades intrínsecas del hombre, no así de la mujer. Su premisa se basaba en la afirmación de que la individualidad y la autonomía eran cualidades propias y exclusivas de los hombres y, por ende, también lo sería la ciudadanía. Esta membresía y adscripción limitada de los hombres a la ciudadanía motivó la imposición de una visión androcéntrica del mundo, al definirse en términos políticos y filosóficos al hombre como modelo sujeto de derechos. En consecuencia, la política se delimitó como un ámbito masculino, con lo cual se justificó la exclusión de las mujeres, bajo el supuesto de la imposibilidad femenina para concebir y discernir ideas y normas morales necesarias para orientar su comportamiento (Pateman, 1995; Canterla, 2002; en Medina y Márquez, 2010: 16).

El debate ilustrado en torno a la igualdad política y la paradójica expulsión de las mujeres del pacto social que dio origen al Estado moderno, motivó la conformación de un movimiento social internacional, conocido como sufragismo, que constituyó la respuesta de las mujeres al monopolio masculino del espacio público. Se trató de la primera acción colectiva organizada por las mujeres, a fin de exigir su estatus de ciudadanas, el cual cobró auge entre las postrimerías del siglo XIX y la primera mitad del siglo XX (Medina y Márquez, 2010: 17 y 18). La exigencia central de este movimiento social fue el derecho de las mujeres a votar y ser electas, además del derecho a la educación y al trabajo remunerado, en concordancia con los principios de la Ilustración en torno a la individualidad, la autonomía como sujetos y la igualdad, en lo que Norberto Bobbio ha escrito como "el derecho a tener derechos" (Medina y Márquez, 2010: 18).

En el sufragismo participaron sucesivas generaciones de mujeres, logrando, apenas de manera difusa en 1948, el reconocimiento internacional de su derecho a participar en el ámbito de la política como un derecho humano fundamental, a través de la Declaración Universal de los Derechos Humanos que establece, en su artículo 21, que "toda persona tiene derecho a participar en el gobierno de su país" (Medina 
y Márquez, 2010: 18). La desigualdad en la participación política de las mujeres ha motivado, desde las últimas décadas del siglo $\mathrm{xx}$, el cuestionamiento internacional acerca del carácter representativo de los gobiernos, así como el reconocimiento de la necesidad de imponer mecanismos que garanticen a mujeres y hombres las mismas oportunidades de participar en la política de forma efectiva y equilibrada, a fin de integrar, de igual manera en la agenda pública, sus visiones, necesidades, intereses y problemas (Medina y Márquez, 2010: 20).

El año 1929 marca el inicio del otorgamiento a las mujeres de un derecho inherente a la ciudadanía en democracia: la posibilidad de participar en la elección de sus gobernantes. Se inicia, así, un proceso de reconocimiento de una lucha que -impulsada por los movimientos de mujeres sufragistas y, en algunos casos, con aliados importantes entre la dirigencia política masculina- comenzó en muchos países en el siglo xix y que tuvo que vencer las resistencias de sectores políticos de diversas ideologías (Llanos y Sample, 2008: 13). Ecuador fue el primer país en reconocer el voto femenino, y debieron pasar más de treinta años para que las mujeres latinoamericanas gozaran de este derecho. Paraguay fue el último país en otorgarlo, en 1961 (Llanos y Sample, 2008: 13).

En 1916 se realiza el Primer Congreso Feminista, impulsado por el general Salvador Alvarado como Gobernador de Yucatán. Uno de los principales acuerdos a los que se llegó en este congreso fue demandar que se otorgara el voto ciudadano a las mujeres (INE). En abril de 1917, después de la promulgación de la Constitución política, se expidió la Ley de Relaciones Familiares, según la cual los hombres y las mujeres tienen derecho a considerarse iguales en el seno del hogar (INE). En 1946, el Partido Acción Nacional presentó la primera iniciativa de reforma el artículo 115 constitucional federal, en donde se proponía que a la mujer se le reconociera y aceptara el voto libre en las elecciones. El 24 de diciembre de ese año, la Cámara de Diputados aprobó la iniciativa enviada por el presidente Miguel Alemán, donde se adicionó al artículo 115 constitucional para que en las elecciones municipales participaran las mujeres en igualdad de condiciones que los varones, con el derecho a votar y ser elegidas, entrando en vigor el 12 de febrero de 1947. Gracias a tal iniciativa, en Aguascalientes, María del Carmen Martín del Campo se convierte en la primera presidente municipal de esa ciudad, y la segunda a nivel nacional.

Fue con Lázaro Cárdenas cuando se lanza la iniciativa de otorgar el voto a las mujeres, quien envió la iniciativa a la Cámara de Senadores;

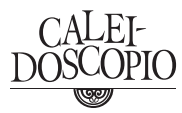


sin embargo, esa promesa se volvió realidad hasta el 17 de octubre de 1953, a iniciativa del presidente electo de la República, Adolfo Ruiz Cortines, al reformarse el artículo 34 constitucional. Pero no fue hasta 1954 que la mujer votó por primera vez en las elecciones y, de igual forma, se reconoció la posibilidad de ser electa. El 17 de octubre de 1953 se publicó en el Diario Oficial el nuevo texto del artículo 34 constitucional: "Son ciudadanos de la República los varones y las mujeres que, teniendo la calidad de mexicanos, reúnan, además, los siguientes requisitos: haber cumplido 18 años, siendo casados, o 21 si no lo son, y tener un modo honesto de vivir" (INE).

Para lograr que las mujeres obtuvieran el voto femenino, se vivió un proceso de casi medio siglo en México, comenzando con la solicitud de Hermila Galindo al Constituyente de 1916-1917, hasta la reforma del artículo 34 constitucional.

Tabla 1.

PROCESO DE EVOLUCIÓN HISTÓRICA DEL RECONOCIMIENTO A LOS DERECHOS POLÍTICOS DE LAS MUIERES EN MÉXICO

\begin{tabular}{|c|c|}
\hline Año & Datos \\
\hline 1917 & $\begin{array}{l}\text { Hermila Galindo solicita al Congreso Constituyente de 1916-1917 el re- } \\
\text { conocimiento de los derechos políticos de las mujeres. }\end{array}$ \\
\hline 1934 & $\begin{array}{l}\text { El presidente Lázaro Cárdenas envía al Congreso de la Unión una inicia- } \\
\text { tiva para reformar el artículo } 34 \text { de la Constitución Política, con el fin de } \\
\text { reconocer la igualdad jurídica de las mujeres y los hombres y, de esta } \\
\text { forma, posibilitar la participación política de las primeras; sin embargo, } \\
\text { la iniciativa no prosperó. }\end{array}$ \\
\hline 1946-1947 & $\begin{array}{l}\text { En 1946, la Cámara de Diputados aprueba la iniciativa en la que se adi- } \\
\text { cionó el artículo } 115 \text { constitucional, que establecía que en las elecciones } \\
\text { municipales participarían las mujeres en igualdad de condiciones que } \\
\text { los varones, con el derecho a votar y ser elegidas. Se publicó en el Diario } \\
\text { Oficial de la Federación hasta 1947, fecha en que entró en vigencia. }\end{array}$ \\
\hline 1953 & $\begin{array}{l}\text { La Cámara de Diputados declara reformados los artículos } 34 \text { y 115, frac- } \\
\text { ción I, de la Constitución Política de los Estados Unidos Mexicanos, con } \\
\text { lo que queda reconocido el voto femenino. }\end{array}$ \\
\hline 1955 & $\begin{array}{l}\text { Las mujeres acuden a las urnas para elegir diputados federales de la } \\
\text { XLIII Legislatura (1955-1958). }\end{array}$ \\
\hline
\end{tabular}

Fragmento de la tabla con el mismo nombre de Peniche C., L. T. y Trejo M., E. (2014). 
En el orden internacional en que se encuentra inmerso el Estado mexicano, en los artículos 3 y 7 de la Convención sobre la eliminación de todas las formas de discriminación contra la mujer (CEDAW, por sus siglas en inglés), impone en cuanto a la participación política de las mujeres y la igualdad de género, dos cuestiones fundamentales: en primera instancia, el reconocimiento del deber de las naciones de garantizar la plenitud de sus derechos y, con ello, el acceso a espacios de toma de decisión; en segunda instancia, la modificación del marco legal y la realización de acciones, incluyendo, desde luego, las estatales, que posibiliten en forma sustantiva, en los hechos, la eliminación de barreras estructurales y culturales generadoras de las asimetrías existentes. De tal forma que México responde con la instauración de acciones afirmativas.

De acuerdo con Alda Facio (2000), las acciones afirmativas parten del reconocimiento de la histórica desigualdad de poder y de goce de derechos entre mujeres y hombres, lo que obliga al Estado a tomar una serie de medidas especiales de carácter temporal, con el objetivo de acelerar el logro de la igualdad entre los sexos sin que éstas constituyan expresiones discriminatorias para los hombres (Medina y Márquez, 2010: 20 y 21). Tales medidas constituyen una disposición concreta en el ámbito internacional que busca dar respuesta al actual desequilibrio de género en los órganos de toma de decisiones (IDEA, 2003: 6, en Medina y Márquez, 2010: 23). Con su implementación se ha buscado hacer de ellas un mecanismo sólido que garantice la efectiva inserción de las mujeres a los organismos de decisión y a las instancias de poder público, a través de la observancia de porcentajes mínimos de participación femenina en los espacios del ámbito de la política (Medina y Márquez, 2010: 23).

Existen tres tipos de cuotas: las adoptadas por la vía constitucional, las cuales se consagran en el ámbito de la ley fundamental de cada país, preferentemente la Constitución; las legislativas, establecidas sobre todo en las leyes electorales; y las que se aplican a las listas de partidos políticos, los cuales utilizan voluntariamente el sistema de cuotas de género en sus procedimientos de selección de cargos internos (Peschard, 1998; Huerta y Magar, 2006: 124, en Medina y Márquez, 2010: 24). Su implementación consiste en la inclusión de un umbral (expresado en un porcentaje) de exigencia mínima que aplica,

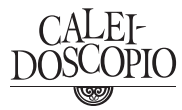


por regla general, en la postulación de candidaturas a un cargo de elección popular; se trata de una medida temporal que se mantiene vigente en tanto logre su objetivo: incrementar el número de mujeres en espacios de toma de decisión, hasta alcanzar la masa crítica deseada (Peña, 2014: 35).

De tal manera, influenciados por la presión de algunas campañas por los derechos de las mujeres, así como la intervención de organismos internacionales, en 1993 se introduce una recomendación al artículo 175 del Código Federal de Instituciones y Procedimientos y Procesos Electorales. La reforma dictaba: "Los partidos políticos, proveerán en los términos que determinen sus documentos internos, una mayor participación de las mujeres en la vida política del país a través de su postulación a cargos de elección popular" (COFIPE, 1993); sin embargo, se debe reiterar que se trata de una recomendación, misma que en las elección de 1994 provocó un crecimiento de 5.3\% (Hevia Rocha, 2015: 13) en cuanto a la llegada de mujeres a las diputaciones, pero no obligaba a los partidos a cumplir con esta reforma. ${ }^{3}$

Gracias a los resultados de la Asamblea Nacional de Mujeres para la Transición Democrática: organizaciones sociales y partidos políticos, en 1996 se dio el paso definitivo a la implementación de cuotas en México con una reforma al artículo 22 transitorio, en el cual se dictaba que los partidos políticos nacionales consideraran en sus estatutos que en las candidaturas legislativas no se excedieran de $70 \%$ para un mismo género (Hevia, 2015: 15), esto para presentar un crecimiento de presencia femenina en los espacios de la legislatura de 3.3\%.

Para el siguiente proceso electoral no hubo reformas al código, por lo cual, se puede especular, fue la razón del descenso de $1.4 \%$ presentado en las elecciones del año 2000, pues de contar con 87 diputadas en la LVII Legislatura, bajó a 80 en la LVIII Legislatura. Lo anterior, aunado al incumplimiento de los estatutos, obligó en la siguiente reforma, la del 2003, a que se estableciera por primera vez un castigo a los partidos que no cumplan con el registro de $70 \%$ de candidatos propietarios del mismo género (Hevia, 2015: 18), logrando un crecimiento de representación femenina de $8 \%$.

3 Como puede observarse, sólo se toma para ejemplificar el impacto a las reformas electorales en cuestión de cuotas de género a la cámara baja del H. Congreso de la Unión. 
Sin noticias nuevas en las elecciones del 2006, en cuanto a reformas al código para la participación femenina, se presenta un nuevo descenso aparentemente inofensivo, pues fue de $0.6 \%$. Se toman nuevas medidas en el 2009, como el aumento porcentual a la cuota, pasando así de un 30/70 a un 40/60, procurando la paridad; se continúa con la negativa de registro al incumplimiento de la cuota, y se aumenta un apartado interesante que dicta el destino de $2 \%$ del financiamiento público para la capacitación, promoción y desarrollo del liderazgo político de las mujeres (Hevia, 2015: 22), lo cual demostró un fuerte crecimiento de $4.6 \%$ en las elecciones del 2009; sin embargo, se definió un nuevo problema, el caso de "las juanitas", que retiró de los curules a 8 diputadas electas. La detección de "las juanitas" en el 2009 llevó al Supremo Tribunal Electoral del Poder Judicial de la Federación a establecer la sentencia 12624, en la que reitera la obligación de los partidos políticos a cumplir con $40 \%$ de espacios destinados a las mujeres, pero bajo la demanda de cubrir las suplencias con personas del mismo género (Peniche y Trejo, 2014; Hevia, 2015). Gracias a la sentencia del Supremo Tribunal Electoral, se logró un aumento de 9\% de diputadas en la LXII Legislatura.

El último paso importante en la participación política de las mujeres encontró su auge en el 2014, cuando tanto en la Ley General de Instituciones y Procedimientos Electorales como en la Ley General de Partidos Políticos, se obligó a los partidos a determinar y hacer públicos los criterios de paridad, así como en las suplencias a aplicar las reglas de paridad; con ello, también se aumentó a 3\% el uso del financiamiento público para la capacitación, promoción y desarrollo del liderazgo político de las mujeres (Peniche y Trejo, 2014; Hevia, 2015).

Es así como de 1993 a 2014 se cumplen 21 años de reformas electorales que hacen necesaria la reforma en la Constitución mexicana en un ámbito de paridad respecto a su participación política; como puede observarse de manera cronológica en la Tabla 2. 
Tabla 2 .

REFORMAS DE LAS CUOTAS DE GÉNERO EN MÉXICO

\begin{tabular}{cl}
\hline Año & \multicolumn{1}{c}{ Reforma } \\
\hline 1993 & $\begin{array}{l}\text { Art. 175, III. Los partidos políticos promoverán en los términos que determinen } \\
\text { sus documentos internos, una mayor participación de las mujeres en la vida } \\
\text { política del país, a través de la postulación a cargos de elección popular. }\end{array}$ \\
\hline 1996 & $\begin{array}{l}\text { Art. } 22 \text { Transitorio. Los partidos políticos nacionales considerarán en sus } \\
\text { estatutos que las candidaturas por ambos principios a diputados y senadores, } \\
\text { no excedan del 70\% para un mismo género. }\end{array}$ \\
\hline
\end{tabular}

2002 Art. 175. Máximo 70\% candidatos propietarios mismo género.

Listas de RP organizadas en segmentos de 3, una candidatura de género distinto.

Negativa de registro si hay incumplimiento.

Excepción de candidaturas de MR resultado de un proceso de elección mediante voto directo.

2008 Arts. 78, 219, 220, 221. 40/60 candidaturas propietarias mismo género, procurando paridad.

Excepción MR resultado proceso de elección democrático.

Listas RP segmentos de 5, dos de género distinto, alternados.

Sanción negativa de registro.

$2 \%$ financiamiento público para capacitación, promoción y desarrollo del liderazgo político de las mujeres.

2011 Candidatos de MR electos mediante voto directo, mínimo 120 y 26 (40\%) candidatos propietarios, mismo género a diputados y senadores, respectivamente.

Candidaturas que conforman la cuota de género, fórmula completa, candidatos mismo género.

2014 Art. 41 Institucional. Los partidos deben establecer las reglas para garantizar la paridad entre los géneros, en candidaturas a legisladores federales y locales.

Segundo Transitorio. Instruye al Congreso a expedir las reglas para garantizar la paridad entre géneros en candidaturas a legisladores federales y locales.

2015 Desarrollo de iniciativas para establecer la paridad en órganos de representación popular federales, estatales y municipales. Compromiso de los partidos y autoridades para garantizarla.

Fuente: elaboración propia con los datos obtenidos de Rocha, T. H. (2015).

60 CENTENARIO DE LA CONSTITUCIÓN 1917-2017 
A partir de la Conferencia de Atenas en 1992 se definió la paridad como la total integración, en pie de igualdad de las mujeres, en las sociedades democráticas, utilizando para ello las estrategias multidisciplinarias que fueran necesarias (Zúñiga, 2005: 1, en Medina y Márquez, 2010: 25). No se trata únicamente de cubrir una cuota mayor de cargos políticos a favor de las mujeres, sino de reconocer y respetar, de manera efectiva y en un sentido amplio, la igualdad entre mujeres y hombres (Medina y Márquez, 2010: 26). Respecto a América Latina, los países de la región establecieron recientemente en el Consenso de Quito (2007) que:

la paridad es uno de los propulsores determinantes de la democracia, cuyo fin es alcanzar la igualdad en el ejercicio del poder, en la toma de decisiones, en los mecanismos de participación y representación social y política, y en las relaciones familiares al interior de los diversos tipos de familias, las relaciones sociales, económicas, políticas y culturales, y que constituye una meta para erradicar la exclusión estructural de las mujeres (CEPAL, 2007: 3, en Medina y Márquez , 2010: 27).

La paridad implica que ningún género tenga más de $40 \%$ de los cargos o, en su versión más radical, establece una distribución de 50\% a $50 \%$. Asimismo, constituye una fórmula que busca superar la idea de una "ciudadanía neutra", a través de la proporcionalidad equitativa de mujeres y hombres en todos los órganos de decisión y representación pública (Medina y Márquez, 2010: 27 y 28). En Latinoamérica, las actitudes hacia la paridad se vuelven cada día más favorables, fortaleciendo y consolidando los avances logrados. El grupo de países integrado por Argentina, Costa Rica y México está en el tercio con mayor porcentaje de mujeres en sus legislaturas a nivel federal (Peniche y Trejo, 2014: 1). Siguiendo con la historia, el 10 de febrero de 2014 se dio paso a la última y actual reforma constitucional, específicamente al artículo 41, fracción I, párrafo segundo, que establece:

Los partidos políticos tienen como fin promover la participación del pueblo en la vida democrática, contribuir a la integración de los órganos de representación política y como organizaciones de ciudadanos, hacer posible el acceso de éstos al ejercicio del poder público, de acuerdo con los

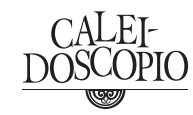


programas, principios e ideas que postulan y mediante el sufragio universal, libre, secreto y directo, así como las reglas para garantizar la paridad entre los géneros, en candidaturas a legisladores federales y locales. Sólo los ciudadanos podrán formar partidos políticos y afiliarse libre e individualmente a ellos; por tanto, queda prohibida la intervención de organizaciones gremiales o con objeto social diferente en la creación de partidos y cualquier forma de afiliación corporativa (CPEUM, art. 41).

Con la reforma, los partidos políticos estarán obligados a postular las reglas necesarias y suficientes para garantizar la paridad entre los géneros (Gobierno de la República), lo que conduce a que congresos locales homologuen ciertas disposiciones en la materia, ya que persisten en algunas entidades federativas expresiones de discriminación hacia las mujeres en el ámbito político-electoral (Peniche y Trejo, 2014: 6). Así, el principio de paridad es exigible para los partidos políticos en las candidaturas a cargos legislativos locales y federales, pero no limita que en la legislación local puedan incluirse cargos ejecutivos (ayuntamientos o regidurías) (INE). Aunque, anterior a la reforma de la Constitución, en 2001, el PRI modificó sus estatutos para exigir paridad de género entre sus candidatos para las posiciones de liderazgo interno y para candidaturas políticas (Baldez, 2012: 164). Ejercicio que están realizando los demás partidos políticos en México para ir de manera armónica con lo que dicta la Constitución Política, así como la normatividad electoral.

\section{CONCLUSIÓN}

A cien años de promulgada la Constitución Política de los Estados Unidos Mexicanos, ésta permite evaluar las trasformaciones en el ámbito político, económico y social. Si bien la Constitución ha sufrido alrededor de 700 modificaciones, ésta ha reaccionado lentamente a las necesidades de la población, como puede observarse al intentar dar respuesta a cuál ha sido la evolución de la participación política de la mujer en México.

Si bien, en el ámbito municipal se veía un área de oportunidad, como se puede observar en el artículo 115 constitucional en 1946, permitiéndole su participación en las elecciones municipales en igualdad de condiciones que los varones, con el derecho a votar y ser elegidas, 
el ingreso formal de la mujer a la vida política del país se da 36 años después a la Constitución de 1917, con el decreto realizado el 17 de octubre de 1953 presentado en el Diario Oficial de la Federación, en el cual se anunciaba que las mujeres podían votar y ser votadas como resultado de la reforma al artículo 34 de la Constitución. Es desde 1993 cuando a partir de la reforma en materia de legislación electoral se intenta promover una mayor participación de la mujer en la vida política, a través de su postulación a cargos de elección popular; a esto se le conoce como acciones afirmativas, cuyo objetivo radica en promover con mayor firmeza su acceso a la política. En este año, por primera ocasión, se observan las acciones afirmativas en México: a manera de recomendación, con el objetivo de una mayor incorporación e inclusión de las mujeres como candidatas a cargos de elección popular, establecida en el art. 175, fracción 3, del Código Federal de Instituciones y Procedimientos Electorales.

Posteriormente, el 22 de noviembre de 1996 se realiza una reforma en donde se establece por primera vez la acción afirmativa como una norma legal, donde se debería contemplar en su norma estatutaria que $70 \%$ de las candidaturas por ambos principios, diputados y senadores, fueran de un mismo género. Es en la reforma del 2002 cuando se establece la acción obligatoria a los partidos políticos de garantizar la participación de las mujeres en sus art. 38, 175, 175-A, 175-B, 175-C. El 14 de enero de 2008 se realizó una nueva reforma como resultado de lo que se vivió en las elección de 2006, ésta consistió en que los partidos y coaliciones debían presentar sus candidaturas con al menos $40 \%$ de candidatos propietarios de un mismo género, y las listas de representación proporcional se integraran por segmentos de cinco candidatos, donde en cada uno de los segmentos de cada lista habría dos candidaturas de género distinto, de manera alternada (ver art. 219, 220, 221 del COFIPE). En el 2011, se menciona que los candidatos propietarios deben ser del mismo género en el caso de diputados y senadores, respectivamente.

Para el 2014 se realiza la última reforma en materia de participación política de la mujer en paridad de condiciones, la cual se puede apreciar en el artículo 41 de la Constitución Política de México, donde menciona que los partidos políticos deben establecer las reglas para garantizar la paridad entre los géneros, en candidaturas a legisladores federales y locales. Ante lo anterior, se puede agregar que la reforma se argumenta no sólo como válida, sino como necesaria ante un total

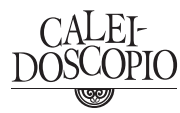


de $83,563,190$ votantes, de los cuales 40, 219,635 son hombres y 43, 343,555 mujeres para el 2015.

\section{REFERENCIAS}

Baldez, L. (2012). Cuotas versus primaria: La nominación de candidatas mujeres en México. Revisado el 10 de febrero de 2018 en: sites.dartmouth.edu/lisabaldez/files/2012/11/Baldez-Cuotasversus-Primarias-2010.pdf.

Facio, A. (2000). El acceso a la justicia desde la perspectiva de género. Recuperado de: http://americalatinagenera.org.org/newsite/images/ cdr-documents/publicaciones/acceso_justicia_facio.pdf.

CeAmeg (2014). Participación Política de las Mujeres 2014. Revisado el 24 de febrero del 2018 en: archivos.diputados.gob.mx/Centros_ Estudio/ceameg/Inv_Finales_08/.../1_19.pdf .

COFIPE (1993). Decreto por el que se reforman, adicionan y derogan diversos artículos del Código Federal de Instituciones y Procedimientos Electorales. Recuperado de: http://diputados.gob. $\mathrm{mx} /$ LeyesBiblio/abro/cofipe.htm.

Girón, A., González M., M. L. y Jiménez, A. V. (2008). Breve historia de la participación política de las mujeres en México. En Límites y desigualdades en el empoderamiento de las mujeres en el PAN, PRI y PRD. Las Ciencias Sociales. Estudios de Género (pp. 33-61). México: Miguel Ángel Porrúa.

Hevia, T. (2015). Evolución y establecimientos de cuotas en México y las implicaciones de la reforma político-electoral de 2014 en cuanto a la aplicación de la partida de género en el registro de candidaturas para la integración de la Cámara de Diputados. México: ine. Recuperado de: https://portalanterior.ine.mx/archivos3/portal/historico/recursos/IFE-v2/DEPPP/DEPPP-Varios/Foro_ ImpactoyProspectivas/docs/terehevia15dic.pdf.

H. Ayuntamiento de Coatzacoalcos. (2018). Conmemoran el Centésimo Primer Aniversario de la Constitución Política de los Estados Unidos Mexicanos. En Gobernantes. Revisado el 17 de febrero del 2018 en: http://www.gobernantes.com/vernota. php?id=207272.

H. Congreso de la Unión. Revisado el 16 de febrero del 2018 en http:// sitl.diputados.gob.mx/LXII_leg/cuadro_genero.php. 
INE. (s/f). Paridad de género: Evolución, logros y realidades. Revisado el 10 de febrero del 2018 en: https://portalanterior.ine.mx/archivos3/portal/historico/recursos/IFE-v2/DEPPP/DEPPP-Varios/Foro_ ImpactoyProspectivas/docs/doraaliciapan29oct.pdf.

Información para votantes (2015). Conoce cuántos ciudadanos en cada estado podrán votar el 7 de junio. Revisado el 17 de febrero del 2018 en: http://www.infoeleccionesmexico.com/informacion-numero-electores-por-estado-94.html.

Llanos, B. y Sample, K. (2008). 30 años de democracia: ¿en la cresta de la ola? Participación política de la mujer en América Latina. Perú: Interntional Institute for Democracy and Electoral Asistance.

López B., L. M. (2017). El peculiar origen de la Constitución de 1917. La Jornada en Línea. Revisado el 17 de febrero del 2018 en: http:// www.jornada.unam.mx/2017/01/29/opinion/012alpol.

Madrid G., M. A. (2016). La Constitución Mexicana de 1917. La Carta Magna que a pesar de sus 99 años aún sigue viva. El Mañanero. Revisado el 17 de febrero del 2018 en: http://diarioelmananero. com.mx/historia/la-constitucion-mexicana-de-1917.

Medina E., A. y Márquez B., R. G. (2010). La participación política de las mujeres. De las cuotas de género a la paridad. México: CEAMEG.

Peniche C., L. T. y Trejo M., E. (2014). La reforma hacia la paridad: cerrando la brecha y promoviendo el liderazgo político de las mujeres. Cámara de Diputados. Comité del Centro de Estudios para el Adelanto de las Mujeres y La Equidad de Género. México: CEAMEG. Revisado el 10 de febrero de 2018 en: http://www3.diputados.gob. mx/camara/content/download/328096/1 158879/files/2014\%2008\%20 Dossier_LPM.pdf.

Peña, B. (2014) La paridad de género: eje de la reforma política_electoral en México. Revista Mexicana de Estudios Electorales, 14, 31-74. Recuperado de: http://www.iepcjalisco.org.mx/sites/default/ files/lectura_de_la_paridad_de_genero.pdf.

Rocha, T. H. (X). Evolución y establecimiento de cuotas en México y las implicaciones de la reforma político-electoral de 2014 en cuanto a la aplicación de la paridad de género en el registro de candidaturas para la integración de la Cámara de Diputados. Revisado el 7 de febrero del 2018 en: http://www.ine.mx/archivos3/portal/historico/ recursos/IFE-v2/DEPPP/DEPPP-Varios/Foro_ImpactoyProspectivas/ docs/terehevia15dic.pdf. 
Salazar U., P. (2015). Sobre el concepto de Constitución. Enciclopedia de Filosofía y Teoría del Derecho (pp. 1930-1958), Vol. 3. México: Universidad Nacional Autónoma de México. Revisado el 17 de febrero del 2018 en: https://archivos.juridicas.unam.mx/www/bjv/ libros/8/3876/9.pdf.

\section{ACERCA DE LOS AUTORES}

Brisa Herminia Campos Aceves (bhcampos@correo.uaa.mx) es maestra en Ciencia Política. Candidata a doctora por la Universidad de Guadalajara. Profesora-investigadora del Departamento de Ciencias Políticas y Administración Pública de la Universidad Autónoma de Aguascalientes (ORCID: 0000-0003-1733-3998).

Juan Pablo Carranza Salas (carranzacontratenor@hotmail.com) es licenciado en Letras Hispánicas por la Universidad Autónoma de Aguascalientes. Asistente en investigación en el Departamento en Ciencias Políticas y Administración Pública de la Universidad Autónoma de Aguascalientes (ORCID: 0000-0002-0166-6125). 